\title{
Loss Distribution of Drought Disasters in Yunnan Province Based on ECM Model
}

\author{
Kaimin $\mathrm{Li}^{*}$, Guang Li' Yuanyuan Zhang \\ Baoshan University, Baoshan Yunnan, 678000, China \\ *Corresponding author.Email: 13170241@qq.com
}

\begin{abstract}
This paper attempts to analyze the relationship between drought disaster area and direct economic loss in Yunnan Province based on the method of co-integration test and the error correction model. Firstly, the stationarity of the sequence is judged according to the auto-correlation and partial auto-correlation analysis diagrams of the sequence and the results of ADF test; Then, the OLS regression model of first-order difference sequence is established. Combined with the residual sequence of OLS estimation and the results of ADF test, it is concluded that the sequence has a co-integration relationship; Finally, the ECM model with error correction term is established. It is found that the model has a good fitting effect, and can well describe the short-term fluctuation and long-term equilibrium relationship between drought disaster affected area and direct monetary loss series.
\end{abstract}

Keywords: Damage area, Direct economic loss, Co-integration test, ECM model.

\section{INTRODUCTION}

Yunnan Province is located in the southeast of China's Qinghai Tibet Plateau. It is located in a special geographical location of the low latitude plateau, with an average altitude of about 2000 meters. It is close to the South China Sea and the Bay of Bengal. Plateau mountains, mountains and valleys, rivers, basins and lakes are scattered everywhere, with many climate types and drastic changes. In particular, several large mountains with approximate north-south trend, represented by Hengduan Mountain, Gaoligong Mountain, nu mountain and Yunling mountain, have blocked the air flow and significantly exacerbated the impact on climate change in Yunnan. Drought disasters in Yunnan occur frequently and last for a long time, and their distribution is consistent. Most regions, especially the central and eastern regions of Yunnan, are most prone to extreme drought [1].

In recent years, serious drought disasters have occurred frequently in Yunnan, and the disaster intensity, duration and impact scope show a gradual upward trend. Peng Guifen et al.[2] used drought disaster index to study the climate characteristics and change trend of drought in Yunnan; Zheng jianmeng et al.[3] studied the extreme meteorological drought index and the climatic characteristics of precipitation and evaporation at drought stations in Yunnan on the basis of comprehensive meteorological drought index analysis method; Li Zhen[4] comprehensively applied the knowledge and methods of multivariate statistical analysis, geographic information system and remote sensing to study the temporal variation and spatial distribution characteristics of main drought influencing factors in Yunnan Province from 1965 to 2011; He Jiaonan et al.[5] used analytic hierarchy process to evaluate the drought affected area and total economic losses in Yunnan Province in recent 10 years. At present, there have been many achievements in the research on the temporal and spatial evolution characteristics, causes and distribution laws of drought disasters. However, with the increase of the demand for global disaster risk management, the importance of the research on the classification and assessment of natural disaster losses has become increasingly prominent. Therefore, it is of great practical significance to study the distribution characteristics of drought disaster losses in Yunnan Province.

\section{ANALYSIS OF DROUGHT DISASTER IN YUNNAN PROVINCE}

The climate of Yunnan Province is complex and diverse. Among them, the three-dimensional climate characteristics are obvious. Drought disasters have occurred frequently since 1996 to 2018 . Affected by the plateau monsoon climate, Yunnan continues to have 
warm winter weather and dry and little rain in spring, with an average annual rainfall of about $1258 \mathrm{~mm}$. The distribution of rainy season and dry season is relatively concentrated. The rainy season is generally from August to October every year, and the effective rainfall accounts for about $85 \%$ of the whole year; The dry season is generally from November to April of the next year (some areas may be delayed to May and June). The rainfall accounts for only about $15 \%$ of the whole year, and the situation of early winter, spring and summer occurs frequently.

In this paper, the regional frequency distribution map of drought disasters in Yunnan Province is drawn based on the drought disaster data from 1996 to 2018 in Yunnan disaster reduction Yearbook.

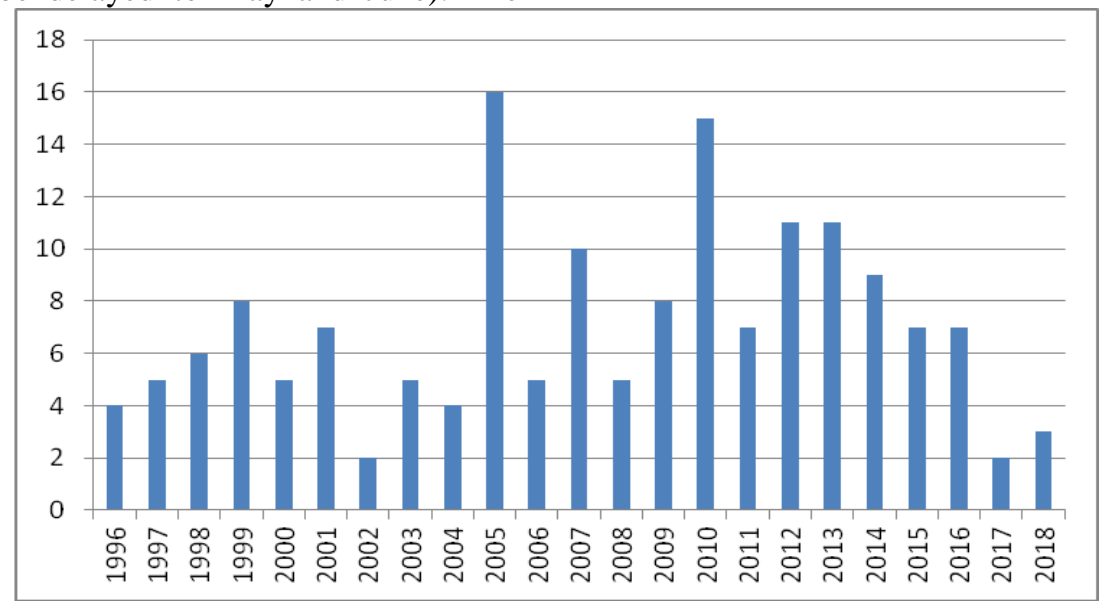

Figure 1 Regional frequency distribution of drought disasters in Yunnan Province from 1996 to 2018

It can be seen from Figure 1 that the number distribution of drought disaster areas in Yunnan Province from 1996 to 2018 is relatively complex. The average number of affected areas in 16 prefectures and cities in Yunnan Province is 7 every year, the mode is 5, the standard deviation is 3.67 , the skewness is 0.962 and the kurtosis is 0.755 . The most serious years are 2005 and 2010. In these five years, 16 prefectures and cities in the province have suffered serious drought disasters, the disaster rate is as high as $70 \%$, and the direct economic loss is more than 20 billion yuan.

In order to deeply understand the impact of drought disaster on regional economy in Yunnan Province, this paper selects the variables closely related to the direct economic loss of drought disaster in Yunnan Province, namely the affected area, disaster area and dead harvest area of drought disaster, to study the fluctuation of drought disaster loss. [6].Data source: Yunnan disaster reduction Yearbook, in which the direct economic loss of drought disaster in Yunnan Province is 100 million yuan; For the unit of measurement of area, since the compilation unit of Yunnan disaster reduction Yearbook is not unified, the unit is 10000 hectares in 2018 and $10000 \mathrm{mu}$ in other years. It has been uniformly converted to $10000 \mathrm{mu}$ in the data sorting stage.

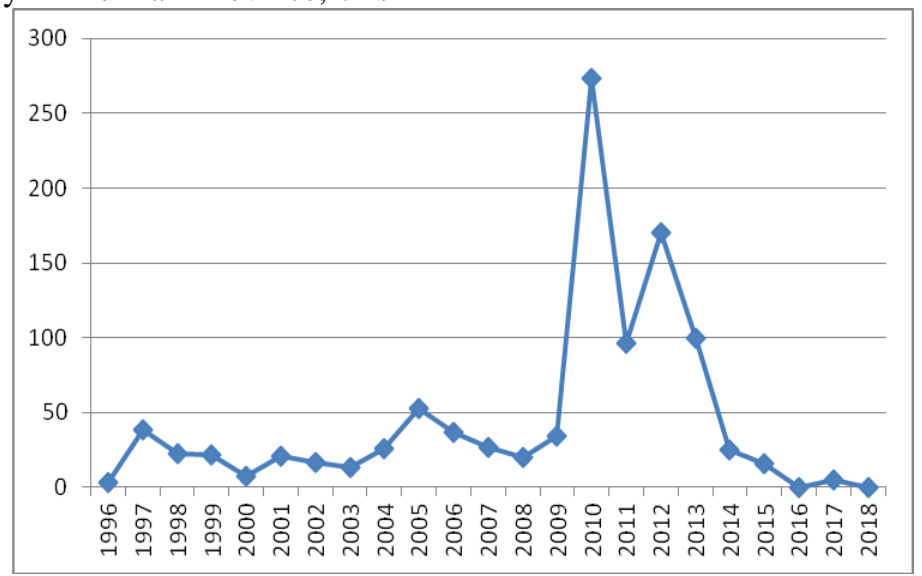

Figure 2 Direct economic loss of drought disaster (100 million yuan) 


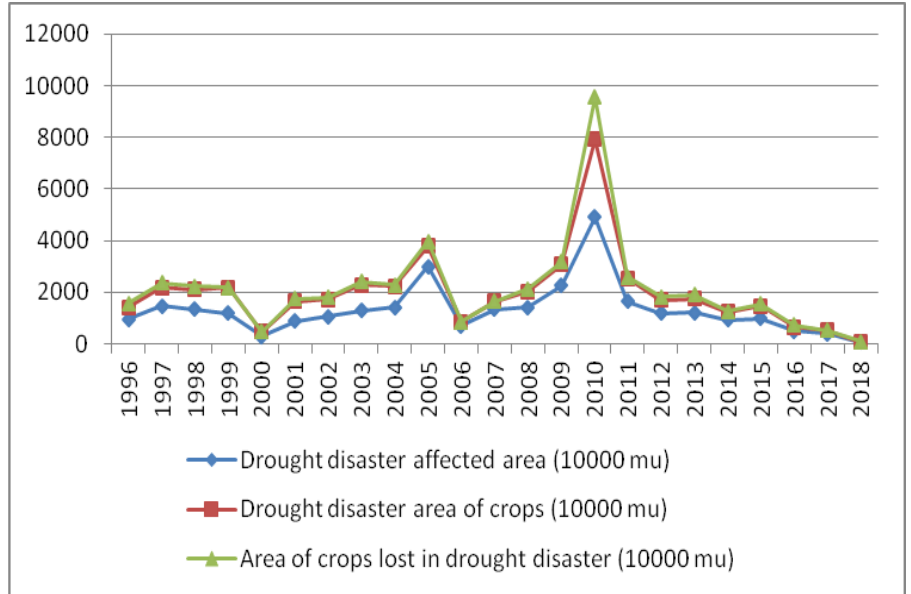

Figure 3 Drought disaster affected area, disaster area and dead harvest area (10000 mu)

It can be seen from figures 2 and 3 that the average annual direct economic loss of drought disaster in Yunnan Province from 1996 to 2018 was about 4.6 billion yuan, with the disaster rate of $30.3 \%$, the disaster rate of $14.9 \%$ and the failure rate of $3.6 \%$, all higher than the national average of $15.0 \%, 8.1 \%$ and $1.7 \%$ [7]. Therefore, drought disaster has become one of the natural disasters with the highest frequency, the widest range of influence and the most serious disaster loss in Yunnan Province.

As can be seen from figures 2 and 3, the annual average direct economic loss caused by drought disasters in Yunnan Province from 1996 to 2018 was about 4.6 billion yuan, with a disaster rate of $30.3 \%$, a disaster rate of $14.9 \%$ and a harvest failure rate of $3.6 \%$. These data are higher than the national average levels of disaster rate, disaster rate and harvest failure rate, which are $15.0 \%, 8.1 \%$ and $1.7 \%$ [7]. Therefore, drought disaster has become one of the natural disasters with the highest frequency, the widest range of influence and the most serious disaster loss in Yunnan Province.

Since the fluctuation trends of drought disaster affected area, disaster affected area and non harvest area in Yunnan Province are roughly the same, researchers can appropriately reduce variables in order to eliminate the multicollinearity of the model and improve the fitting effect of the model.

Table 1. Pearson correlation coefficient between drought disaster area and direct economic loss

\begin{tabular}{c|cccc}
\hline & $\begin{array}{c}\text { Direct } \\
\text { economic loss }\end{array}$ & $\begin{array}{c}\text { Affected } \\
\text { area }\end{array}$ & Disaster area & $\begin{array}{c}\text { Dead harvest } \\
\text { area }\end{array}$ \\
\hline $\begin{array}{c}\text { Direct } \\
\text { economic loss }\end{array}$ & 1 & 0.731 & 0.735 & 0.798 \\
Affected area & 0.731 & 1 & 0.889 & 0.831 \\
Disaster area & 0.735 & 0.889 & 1 & 0.916 \\
Dead harvest a & 0.798 & 0.831 & 0.916 & 1 \\
rea & & & & \\
\hline
\end{tabular}

According to the Pearson correlation coefficient between drought disaster area and direct economic loss in Yunnan Province, the correlation coefficient between drought disaster direct economic loss and the other three variables is more than 0.7 , while the correlation coefficient of disaster area, disaster area and dead harvest area has exceeded 0.8 . In order to reduce the multicollinearity of the model, it is considered to eliminate the disaster area the three variables, disaster area and harvest area, eliminate the information overlap caused by too many explanatory variables.

Due to the dimensional difference between the original data of dependent variables and independent variables, it is easy to have the problem of pseudo regression when directly establishing the measurement model. Therefore, this paper uses the standardized data to establish OLS regression model. Taking the direct economic loss of drought disaster in Yunnan Province 
as the dependent variable $\left({ }^{y}\right)$ and the affected area as the independent variable $(x)$, the regression OLS model is established. The goodness of fit of the model is $R^{2}=0.534$, and the fitting effect is not very ideal. The classical OLS model is used to describe the relationship between dependent variables and independent variables, ignoring some information contained in the variables themselves, so the model is rough. Combined with figure 2 and figure 3 , it is not difficult to see that although the fluctuations of $x$ and $y$ are uncertain, the changes of the two sequences have certain regularity. Next, consider using the time series model to study the relationship between direct economic loss and affected area.

\section{STABILITY TEST OF DROUGHT DISASTER AFFECTED AREA AND DIRECT ECONOMIC LOSS SERIES IN YUNNAN PROVINCE}

To study the random fluctuation characteristics of direct economic loss series and disaster area series, it is usually necessary to assume that the time series is a stationary white noise series. That is, for sequences $\left\{X_{t}\right\}(t=1,2, \cdots)$, If $E\left(X_{t}\right)=\mu, \quad D\left(X_{t}\right)=\sigma^{2}$ are constants independent of time $t$, covariance $\operatorname{Cov}\left(X_{t} X_{t+k}\right)=\gamma_{k}$ is a constant related only to the time interval $k$, it has nothing to do with time $t$, the time series is said to be wide and stable. The precondition of establishing time series model is that the time series is required to be stationary. Therefore, it is necessary to test the stationarity of time series first.

\subsection{Auto-correlation and partial} auto-correlation analysis of drought disaster affected area and direct economic loss series in Yunnan Province

Generally, we can use the auto-correlation and partial auto-correlation analysis diagram of time series to judge the stationarity of time series.

\begin{tabular}{|c|c|c|c|c|c|c|c|c|}
\hline \multicolumn{2}{|c|}{ Autocorrelation } & \multicolumn{2}{|c|}{ Partial Correlation } & & $A C$ & PAC & Q-Stat & Prob \\
\hline 1 & اتص & $E$ & יاב & 1 & 0.367 & 0.367 & 3.5187 & 0.061 \\
\hline 1 & 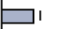 & 1 & コ & 2 & 0.341 & 0.238 & 6.7000 & 0.035 \\
\hline 1 & b 1 & 10 & 1 & 3 & 0.059 & -0.151 & 6.8008 & 0.079 \\
\hline $1 \sqsubset$ & 1 & 1 구의 & 1 & 4 & -0.164 & -0.279 & 7.6103 & 0.107 \\
\hline 1 & 1 & 1 & 1 & 5 & -0.164 & -0.023 & 8.4694 & 0.132 \\
\hline $1 \sqsubset$ & 1 & 1 & 1 & 6 & -0.221 & -0.019 & 10.115 & 0.120 \\
\hline 1 & 1 & 1 & 1 & 7 & -0.162 & -0.035 & 11.057 & 0.136 \\
\hline 15 & 1 & 1 - & I & 8 & -0.175 & -0.126 & 12.232 & 0.141 \\
\hline 1[ & 1 & 1 & 1 & 9 & -0.097 & -0.021 & 12.615 & 0.181 \\
\hline 15 & 1 & 10 & 1 & 10 & -0.130 & -0.098 & 13.367 & 0.204 \\
\hline 1[ & 1 & t & 1 & 11 & -0.100 & -0.082 & 13.846 & 0.242 \\
\hline 1[ & 1 & c & 1 & 12 & -0.100 & -0.082 & 14.373 & 0.278 \\
\hline
\end{tabular}

Figure 4 Auto-correlation and partial auto-correlation analysis of sequence $y$

\begin{tabular}{|c|c|c|c|c|c|c|c|c|}
\hline \multicolumn{2}{|c|}{ Autocorrelation } & \multicolumn{2}{|c|}{ Partial Correlation } & & $A C$ & PAC & Q-Stat & Prob \\
\hline 1 & • & 1 & घ & 1 & 0.305 & 0.305 & 2.4286 & 0.119 \\
\hline 10 & 1 & 1 & 1 & 2 & 0.095 & 0.002 & 2.6732 & 0.263 \\
\hline 1 & 1 & 1 & 1 & & -0.024 & -0.059 & 2.6904 & 0.442 \\
\hline d & 1 & 1 & I & & -0.090 & -0.073 & 2.9359 & 0.569 \\
\hline E & I & 10 & $コ$ । & 5 & 0.136 & 0.211 & 3.5297 & 0.619 \\
\hline 10 & 1 & 16 & 1 & & -0.095 & -0.218 & 3.8336 & 0.699 \\
\hline 1 든 & 1 & 1 든 & 1 & & -0.224 & -0.192 & 5.6383 & 0.583 \\
\hline 18 & 1 & 1 日 & 1 & & -0.306 & -0.193 & 9.2198 & 0.324 \\
\hline 1 口 & 1 & 1 & 1 & 9 & -0.234 & -0.039 & 11.464 & 0.245 \\
\hline 10 & 1 & 1 마 & 1 & 10 & -0.177 & -0.198 & 12.851 & 0.232 \\
\hline 17 & 1 & 17 & 1 & 11 & -0.071 & 0.014 & 13.092 & 0.287 \\
\hline 1 & 1 & 1 & 1 & 12 & -0.018 & 0.002 & 13.109 & 0.361 \\
\hline
\end{tabular}

Figure 5 Auto-correlation and partial auto-correlation analysis of sequence $\mathrm{x}$

It can be seen from Figures 4 and 5 that the values of auto-correlation coefficient (AC) and partial auto-correlation coefficient (PAC) of direct economic loss sequence $y$ and drought disaster affected area $x$ quickly tend to 0 after $k=2$. Therefore, sequence $y$ has second-order auto-correlation and first-order partial auto-correlation, and sequence $x$ has first-order auto-correlation and partial auto-correlation.

\subsection{ADF test of drought disaster affected area and direct economic loss sequence in Yunnan Province}

By observing the distribution of $\mathrm{AC}$ value and PAC value in the auto-correlation analysis diagram and partial auto-correlation analysis diagram of time series, this method to judge the stability of the series is relatively rough, which may misjudge the order of $A R(p)$. Using the auto-regressive model of time variable $t$ to construct ADF test statistics is a more rigorous method to test the stationarity of time series.

It is assumed that sequence $y$ obeys $A R(p)$ process:

$y_{t}=\rho_{1} y_{t-1}+\rho_{2} y_{t-2}+\mathrm{L}+\rho_{p} y_{t-p}+\varepsilon_{t}$

where $\varepsilon_{t}$ is white noise.

Rewrite (1):

$$
\nabla y_{t}=\gamma y_{t-1}+\xi_{1} \nabla y_{t-1}+\xi_{2} \nabla y_{t-2}+\mathrm{L}+\xi_{p-1} \nabla y_{t-p+1}+\varepsilon_{t}
$$

In equation (2) $\gamma=\rho_{1}-1$,

The given test hypothesis is: $H_{0}: \gamma=0, H_{1}: \gamma<0$

Dickey and fuller give the simulation critical value of ADF statistics under the original assumption that the sequence has a unit root [8].

It can be seen from Figures 4 and 5 that the direct economic loss sequence $y$ of drought disaster in Yunnan Province and the affected area sequence $x$ of drought disaster in Yunnan Province are $A R(1)$ 
processes. Firstly, ADF test is used to test the stationarity of sequences $y$ and $x$; If the sequence is non-stationary, the first-order phase by phase difference method is used to eliminate the random trend of the sequence. The sequence after the first-order difference is recorded as $l y$ and $l x$ respectively, the results of ADF inspection are shown in Table 2:

Table 2. ADF test results of each sequence

\begin{tabular}{c|cccccc}
\hline variable & $\begin{array}{c}\text { ADF } \\
\text { statistics }\end{array}$ & $\begin{array}{c}\text { Ending } \\
\text { probability }\end{array}$ & $1 \%$ threshold & $5 \%$ threshold & $10 \%$ threshold & conclusion \\
\hline$y$ & -3.094968 & 0.0417 & -3.769597 & -3.004861 & -2.642242 & Nonstationary \\
$x$ & -3.050107 & 0.0457 & -3.769597 & -3.004861 & -2.642242 & Nonstationary \\
$l y$ & -7.436739 & 0.0000 & -3.788030 & -3.012363 & -2.646119 & stationary \\
$l x$ & -6.317907 & 0.0000 & -3.788030 & -3.012363 & -2.646119 & stationary \\
\hline
\end{tabular}

In Table 2, The value of $t$ statistic in the ADF test result of sequence $y$ is -3.094968 , and the value of $t$ statistic in the ADF test result of sequence $x$ is -3.050107 . Given that the significance level is $1 \%$, the ADF statistics of series $x$ and $y$ are greater than the critical value of the statistics. The original hypothesis is not rejected, that is, both series $x$ and $y$ have unit roots and are non-stationary time series, so the series needs to be adjusted.

After the first-order phase by phase difference, the value of $t$ statistic in the ADF test result of sequence $l y$ is -7.414439 , and the value of $t$ statistic in the $\mathrm{ADF}$ test result of sequence $l x$ is -6.317907 . Given that the significance level is $1 \%$, the ADF statistics of sequenc es $x$ and $y$ are less than the critical value of $t$ statistics. The original assumption should be rejected, that is, the sequences $l y$ and $l x$ after the first-order difference have no unit roots and are stationary time series.

\section{ERROR CORRECTION MODEL OF DROUGHT DISASTER AFFECTED AREA AND DIRECT ECONOMIC LOSS SERIES IN YUNNAN PROVINCE}

$$
\text { If random process }\left\{y_{t}, t=1,2, \mathrm{~L}\right\}
$$

$$
y_{t}=\rho y_{t-1}+\varepsilon_{t}
$$

Where $\rho=1, \varepsilon_{t}$ is a stable process, even $E\left(\varepsilon_{t}\right)=0 \quad, \quad \operatorname{Cov}\left(\varepsilon_{t}, \varepsilon_{t-1}\right)=\mu_{t}<\infty \quad$, $s=0,1,2, \mathrm{~L}$, then the stochastic process satisfying the above conditions is called a unit root process. If a unit root process becomes a stationary process after $d$-order difference, that is
$y_{t}-y_{t-1}=(1-B) y_{t}=\varepsilon_{t}$, such a time series $y_{t}$ is called a $d$-order single integer series.

Because the original time series in this paper are non-stationary time series, and the series after first-order phase-by-phase difference are stationary series, according to the definition of $d$-order single integer series, the original series is first-order single integer series $I(1)$, which meets the premise of co-integration test.

\subsection{Co-integration test of drought disaster affected area and direct economic loss series in Yunnan Province}

In this paper, the EG two-part test method proposed by Engle and Granger is used to test the co-integration relationship between two variables $x_{t}$ and $y_{t}$ :

In the first step, the OLS regression model is established for the sequence $x_{t}$ and $y_{t}$ of $d$-order single integer with the same order, and the estimation error is calculated, that is:

$$
y_{t}=\alpha+\beta x_{t}+e_{t}
$$

The OLS estimation equation is $\hat{y}_{t}=\hat{\alpha}+\hat{\beta} x_{t}$ : where $\hat{\alpha}$ and $\hat{\beta}$ represent the estimated values of regression coefficients $\alpha$ and $\beta$. The estimated residual value of the defined model is :

$$
\hat{e}_{t}=y_{t}-\hat{\alpha}+\hat{\beta} x_{t}
$$

The second step is to test the monotonicity of $\hat{e}_{t}$ series. If $\hat{e}_{t}$ is a stationary series $\hat{e}_{t} \square I(0)$, there is a 
co-integration relationship between variables $x_{t}$ and $y_{t}$, which is called $(1,-\hat{\beta})$ as the co-integration vector, and formula (4) is called the co-integration regression equation.
In this paper, firstly, the OLS regression model is established by using the sequence $l y$ of first-order phase by phase difference to $l x$. The estimation results of the model are as follows:

Table 3. Ordinary least squares estimation results of $l y$ for $l x$

\begin{tabular}{c|cccc}
\hline variable & $\begin{array}{c}\text { Parameter } \\
\text { estimate }\end{array}$ & $\begin{array}{c}\text { Sample standard deviation of } \\
\text { estimator }\end{array}$ & t- statistic & $\begin{array}{c}\text { t- Ending probability of } \\
\text { Statistics }\end{array}$ \\
\hline$b x$ & 0.727900 & 0.144299 & 5.044401 & 0.0001 \\
\hline
\end{tabular}

According to table 3, the goodness of fit of OLS model is 0.547861, and the fitting effect is general. Next, ADF test is performed on the residual sequence $\hat{e}_{t}$ of OLS regression model, and the results are as follows:

Table 4. Unit root test results of residual sequence

\begin{tabular}{c|cccccc}
\hline variable & $\begin{array}{c}\text { ADF } \\
\text { statistics }\end{array}$ & $\begin{array}{c}\text { Ending } \\
\text { probability }\end{array}$ & $1 \%$ threshold & $5 \%$ threshold & $10 \%$ threshold conclusion & contionary \\
\hline$e$ & -6.697193 & 0.0000 & -3.788030 & -3.004861 & -2.646119 & station \\
\hline
\end{tabular}

It can be seen from table 4 that the value of ADF test statistic $t$ of the residual sequence is -6.697193 , which is less than the critical value of $t$ statistic -3.788030 when the given significance level is 0.01 . The original hypothesis is not rejected. It is considered that the residual sequence $\hat{e}_{t}$ is a stationary sequence. It can be concluded from the EG two-part test that the sequence $l y$ and $l x$ have a co-integration relationship.

\subsection{Error correction model of drought disaster affected area and direct economic loss series in Yunnan Province}

In the process of establishing time series analysis model, non-stationary time series are usually transformed into stationary time series by $d$-order phase-by-phase difference method, and then the differential regression model is established:

$$
\Delta y_{t}=\alpha \Delta x_{t}+v_{t}
$$

However, doing so will lead to two problems: first, if there is a long-term stable equilibrium relationship between $x_{t}$ and $y_{t}$ and the error term sequence of the regression model is not correlated, then $v_{t}$ in equation (6) has sequence correlation and is a first-order moving average sequence; Second, the differential regression model is directly established for parameter estimation, which may ignore some important information about the level value of variables
${ }^{[10]}$. Therefore, this paper selects error correction model (ECM) to study the relationship between drought disaster affected area and direct economic loss series in Yunnan Province. Set the ECM model as:

$$
\nabla y_{t}=\beta_{0}+\beta_{1} \nabla x_{t}+\lambda e c m_{t-1}+\varepsilon_{t}
$$

Where ecm is the error correction term. (7) the formula can be explained by the distributed lag model $\operatorname{ADL}(1,1)$ :

$$
y_{t}=\beta_{0}+\beta_{1} x_{t}+\beta_{2} y_{t-1}+\beta_{3} x_{t-1}+\varepsilon_{t}
$$

The following results can be obtained by moving items:

$$
\nabla y_{t}=\beta_{0}+\beta_{1} \nabla x_{t}-\left(1-\beta_{2}\right)\left[y_{t-1}-\frac{\beta_{1}+\beta_{3}}{1-\beta_{2}} x_{t-1}\right]+\varepsilon_{t}
$$

Obviously, equation (9) is the equivalent form of error correction model (7), in which ecm $=y-\frac{\beta_{1}+\beta_{3}}{1-\beta_{2}} x$. (9) the formula clearly reflects the factors affecting the short-term fluctuation $\nabla y_{t}$ of dependent variable $y_{t}$ : first, the influence from the short-term fluctuation $\nabla x_{t}$ of independent variable; The second is from the error correction term ecm. If there is a long-term equilibrium relationship between the two variables $y_{t}$ and $x_{t}$, that is, $\bar{y}=a \bar{x}$ the term ecm in equation (7) can be rewritten as: 


$$
\bar{y}=\frac{\beta_{1}-\beta_{2}}{1-\beta_{2}} \bar{x}
$$

It shows that $\mathrm{ecm}$ can reflect the deviation degree between short-term fluctuation and long-term equilibrium of variable series, so $\mathrm{ecm}$ is also called equilibrium error.

In model (8) $\left|\beta_{2}\right|<1$, therefore, corresponds to $\lambda=-\left(1-\beta_{2}\right)<0$ in equation (7); Moreover, if $y_{t}$ and $x_{t}$ are both first-order single integer sequences, then $\nabla y_{t}$ and $\nabla x_{t}$ in equation (7) are stationary sequences. In this paper, the sequences $y_{t}$ and $x_{t}$ are single integer sequences, and ecm $=\frac{\beta_{1}-\beta_{2}}{1-\beta_{2}}$ is co-integration coefficient.

According to the relationship between co-integration test and error correction model, an error correction model is established:

Step 1: the ordinary least squares (OLS) method is used for co-integration regression. According to the stationarity of the residual sequence in the regression model, judge whether there is a co-integration relationship between the original variables, and give the estimation of co-integration vector $(1,-\hat{\beta})$.
Step 2: if the co-integration relationship between variables exists, the residual of $y_{t}=\alpha+\beta x_{t}+e_{t}$ model is added to the model $\nabla y_{t}=\beta_{0}+\beta_{1} \nabla x_{t}+\lambda e c m_{t-1}+\varepsilon_{t}$ as the unbalanced error term ecm, and the corresponding parameters of ECM model are estimated by ordinary least square method again.

In 4.1, it has been proved that there is a co-integration relationship between sequence $l y$ and $l x$, so the error correction model between drought disaster affected area $l x$ and direct economic loss sequence $l y$ in Yunnan Province can be established.

Firstly, the error correction term is defined according to the results of the least squares estimation in 4.1

$$
e c m=l y_{t-1}-c_{0} l x_{t-1}=l y_{t-1}-0.7279 l x_{t-1}
$$

Then, the ordinary least square method is used to estimate the corresponding parameters in the error correction model:

$$
l y_{t}-l y_{t-1}=c_{1}\left(l x_{t}-l x_{t-1}\right)+c_{2}\left(l y_{t-1}-c_{3} l x_{t-1}\right)+\varepsilon_{t}
$$

Table 5. ECM estimation and related inspection results

\begin{tabular}{c|cccc}
\hline variable & $\begin{array}{c}\text { Parameter e } \\
\text { stimate }\end{array}$ & $\begin{array}{c}\text { Sample standard } \\
\text { deviation of estimator }\end{array}$ & $\begin{array}{c}\text { t- statisti } \\
\mathrm{c}\end{array}$ & $\begin{array}{c}\text { t- Ending probability of } \\
\text { Statistics }\end{array}$ \\
\hline$d(l x)$ & 0.709715 & 0.085155 & 8.334424 & 0.0000 \\
$e c m$ & -1.410085 & 0.211313 & -6.672979 & 0.0000 \\
\hline
\end{tabular}

According to table 5, the error correction model of drought disaster affected area $l x$ and direct economic loss sequence $l y$ in Yunnan Province is as follows:
Where $\lambda=-1.410085<0$ meets the requirements of error correction model.

$l y_{t}-l y_{t-1}=0.709715\left(l x_{t}-l x_{t-1}\right)-1.410085\left(l y_{t-1}-0.7279 l x_{t-1}\right)+\varepsilon_{t}$

Table 6. Comparison results of effectiveness between OLS model and ECM model of $l y$ to $l x$

\begin{tabular}{c|ccc}
\hline & Goodness of fit $\mathrm{R}^{2}$ & AIC value & SC value \\
\hline OLS model & 0.547861 & 2.372608 & 2.422201 \\
ECM model & 0.872617 & 2.334767 & 2.434245 \\
\hline
\end{tabular}

According to the comparative analysis in Table 6, the goodness of fit of OLS model and ECM model of drought disaster affected area $l x$ and direct economic 
loss sequence $l y$ in Yunnan Province is more than 0.5, of which the goodness of fit of ECM model is 0.872617 , which is significantly improved compared with OLS model. At the same time, the AIC value and SC value of ECM model are smaller than those of OLS model. Therefore, it is reasonable to select ECM model.

\section{CONCLUSIONS}

By analyzing the regional frequency distribution of drought disasters in Yunnan Province from 1996 to 2018, it is found that the impact range of drought disasters in Yunnan Province is relatively wide, the average annual drought disaster area is more than 7 prefectures and cities, and the duration is long, and the disaster rate and harvest failure rate are high. This situation has seriously affected the development of the regional economy. Taking the drought disaster area of Yunnan Province as the independent variable and the direct economic loss as the dependent variable, this study further analyzes the correlation between the two variables. Firstly, the stability of the sequence is judged based on the auto-correlation analysis diagram and partial auto-correlation analysis diagram of the sequence and the results of ADF test. Because the time series is non-stationary, researchers must make a first-order phase-by-phase difference to eliminate the non-stationary of the series; then, the OLS regression model is established by using the time series after the first-order difference. Since the sequence after the first-order difference is a stationary sequence, and the residual sequence estimated by OLS is also a stationary sequence, it can be established that there is a co-integration relationship between variables. Therefore, this study further establishes the ECM positive model with error correction term. According to the estimated results of the model, the error correction model of drought disaster affected area and direct economic loss series in Yunnan Province, which has a good fitting effect, and can well describe the short-term fluctuation and long-term equilibrium relationship between drought disaster affected area and direct economic loss series.

\section{ACKNOWLEDGMENTS}

About the author: Li Kaimin (1987), female, from Baoshan, Yunnan Province, lecturer, research direction: risk management. Fund Project: jointspecial project of local colleges and Universities - Youth Project: Research onInfluencing Factors and Loss Measurement of Drought Disaster Risk in Yunnan Province (202001BA070001-122)

\section{REFERENCES}

[1] Zhang Wancheng, Zheng jianmeng, Ren Juzhang. Characteristic Analysis of Extreme Climate Drought in Yunnan [J]. Disaster Science, 2013, 28 (01): 59-64. (In Chinese)

[2] Peng Guifen, Liu Yu, Zhang Yiping. Study on Climate Characteristics and Change Trend of Drought in Yunnan [J]. Disaster Science, 2009, 24(04):40-44. (In Chinese)

[3] Zheng Jianmeng, Huang Wei, Chen Yan, Zhou Jianqin. Study on Extreme Meteorological Drought Indicators in Yunnan [J]. Plateau Meteorology, 2017, 36 (04): 1039-1051. (In Chinese)

[4] Li Zhen. Study on Temporal and Spatial Characteristics of Drought in Yunnan Province [D]. Kunming University of Technology, 2014. (In Chinese)

[5] He Jiaonan, Li Yungang, Li Xue, Huang Jiangcheng. Drought Disaster Risk Assessment in Yunnan Province [J]. Journal of Natural Disasters, 2016, 25 (05): 37-45. (In Chinese)

[6] Han Lanying. Study on Disaster Causing Factors, Risk Characteristics and Impact Mechanism of Agricultural Drought Disaster in China under the Background of Climate Warming [D]. Lanzhou University, 2016. (In Chinese)

[7] Han Lanying, Zhang Qiang, Zhao Hongyan, Huang Tao, Jia Jianying, Zhang Xudong. Loss Characteristics of Agricultural Drought Disaster in Gansu Province and its Response to Climate Warming [J]. China Desert, 2016, 36 (03): 767-776. (In Chinese)

[8] Liu Yujiao, LV Yuhua. Empirical Analysis of VAR Model Based on Non-Stationary Time Series [J]. Journal of Qufu Normal University (NATURAL SCIENCE EDITION), 2021, 47 (01): 41-46 + 51 . (In Chinese)

[9] Li Zinai, Ye Azhong. Advanced Econometrics [M]. Beijing: Tsinghua University Press, 2012:88-89. (In Chinese)

[10] Lin Aihua, Shen Lisheng. Identification of Error Correction Coefficient in Vector Error Correction Model [J]. Statistics and Decision Making, 2020, 36 (08): 27-31. (In Chinese) 